\title{
Post-Process CMOS Front End Engineering With Focused Ion Beams
}

\author{
A. Lugstein ${ }^{1}$, W. Brezna ${ }^{1}$, B. Goebel ${ }^{2}$, L. Palmetshofer ${ }^{3}$, and E. Bertagnolli ${ }^{1}$ \\ 1) Vienna University of Technology, Floragasse 7, 1040 Vienna, Austria \\ ${ }^{2)}$ Infineon Technologies AG Manfred von Ardenne Ring 20 Haus $E$ \\ 3) Johannes Kepler Universität Linz, Altenbergerstr. 69A, 4040 Linz
}

Phone: 0043158801362 41; FAX: 0043158801362 91; E-mail: alois.lugstein@tuwien.ac.at

\begin{abstract}
For the first time we demonstrate the capability of focused ion beams for engineering in the front end regime. We have shown that by a robust protection for MOSFET, Focused ion beam induced ESD degradation can be avoided and implantation induced damage could be well controlled and mitigated by a moderate RTA process. By a masked through gate implantation technique followed by a moderate annealing/activation process we have exemplarily modified the threshold voltage of test transistors on fully featured wafers whereby any unintentional change of devices nearby could be avoided.
\end{abstract}

\section{Introduction}

Focused Ion Beam (FIB) technology has become a critical enabler of rapid prototyping production [1], helping to get new IC devices to market faster. Especially for analog and mixed-signal ICs, the synergy of FIB and mechanical microprobing results in a shortening and a simplification of the debug process [2]. Thereby the FIB is used to expose buried tracks for ebeam or mechanical probing, and to cut tracks and to make new connections which allow design modifications to be verified before new silicon is manufactured. To respond to the demand for FIB operations of complex ICs a number of guidelines and techniques have been presented that concurrently eliminates the possibility of damaging circuit functionality during modification. Up to now circuit modifications are, however, restricted to the back end of the process due to FIB related device degradation [3].

With the focused ion beam system most of the microfabrication steps used in the ICs processing can be performed locally without the use of mask or resist. Expanding FIB operations to the front end regime the unprecedented flexibility of modern FIB systems would allow for example the fabrication of transistors with different channel doping concentrations on the same die. This kind of very customized processing can be considered particularly for the fabrication of some high performance devices in limited amount on the same die, side by side with devices fabricated cheaply by conventional IC technology.
The purpose of this work is to explore the FIB as a high resolution restructuring tool for front end prototyping.

\section{Experimental Techniques}

All experiments were conducted with a Micrion 2500 FIB system at $50 \mathrm{kV}$ acceleration voltage and a beam current of $45 \mathrm{pA}$. The proposed method is demonstrated relying on preprocessed, fully featured CMOS devices with $0.4 \mu \mathrm{m}$ planar silicon gate technology, incorporating LDD and a gate oxide thickness of $9 \mathrm{~nm}$. The dies were arranged on a special 4 terminal sample holder that enables electrically sensing respectively biasing of the device under test (DUT) during the whole beam exposure. In order to avoid interfering geometrical effects, rather large devices were investigated incorporating a channel length of $0.6 \mu \mathrm{m}$ for $\mathrm{p}-\mathrm{MOS}$ and $0.75 \mu \mathrm{m}$ for $\mathrm{n}$-MOS devices and a channel width of 10 $\mu \mathrm{m}$ for both types. As shown in Fig. 1, a milling window was designed to $0.2 \mu \mathrm{m} \times 12 \mu \mathrm{m}$ placed in the center of the device channel and running along the full transistor width. During milling through the passivation and further in the underlying gate the window transforms to a $\mathrm{V}$-shaped trench with footprints of about $30 \mathrm{~nm}$.

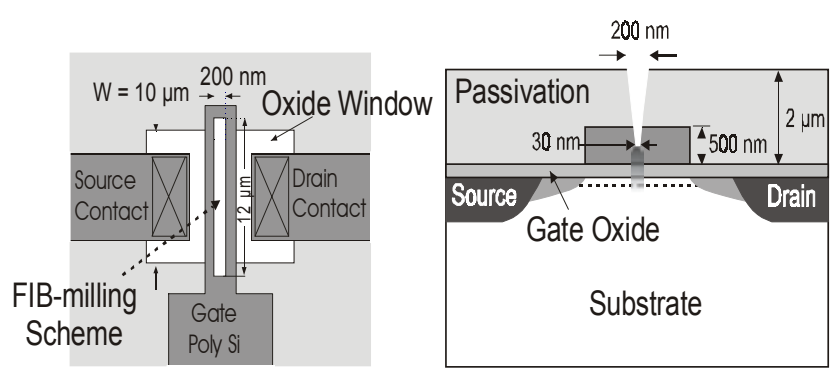

Figure 1. Schematic layout and cross sectional view of the DUT. The FIB milling window is located in the center of the gate.

Cross sectional TEM images of FIB prepared reference devices on the same die were employed for milling depth control. After deep trench milling the devices were subjected to gallium and phosphorus broad beam implantation, whereby the energies were chosen to place the concentration maximum of the doping slightly below the gate dielectric (Tab. 1). 
Finally rapid thermal annealing $\left(600^{\circ} \mathrm{C}\right.$ for $\left.40 \mathrm{~s}\right)$ in forming gas was applied to anneal the implantation damage and activate the dopants, yielding narrow $\mathrm{p}^{+}$, respectively $\mathrm{n}^{+}$regions in the channel. Electrical characterizations were performed before FIB exposure, during FIB milling and finally after RTA thus monitoring the device characteristics during full processing. The influence of FIB processing, through gate implantation and subsequent annealing/activation on the interface state density was obtained by a standard charge pumping technique [4]

\section{Electrical Results and Discussion}

When the ion beam strikes the fully featured wafer, $\mathrm{Ga}^{+}$ ions are implanted in the passivation layer. In combination with the extraction of secondary electrons the deposited positive charge modifies the physical properties of the dielectric layer, while a leakage current appears and changes the performance of underlying transistors [5]. Milling a $1.6 \mu \mathrm{m}$ deep trench as depicted in Fig. 1 leads to a significant increase of the off-state leakage current accompanied by a stretchout of the threshold voltage (Fig. 2). Such $\mathrm{V}_{\text {th }}$ shift is associated with the creation of amphoteric interface traps that capture majority carriers during post-irradiation testing. As a result, both types of transistors move further into enhancement mode and the absolute value of $\mathrm{V}_{\text {th }}$ increases, as observed.

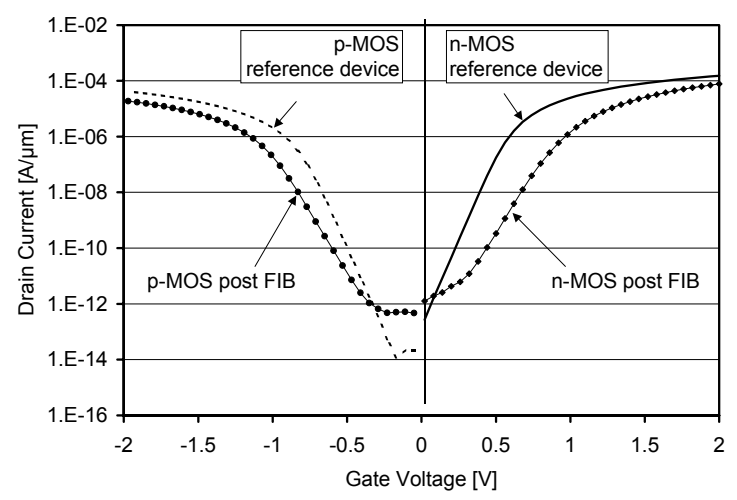

Figure 2. Comparison of the transfer characteristics of MOS devices before and after FIB exposure of the floating device.

The maximum threshold shift found was $520 \mathrm{mV}$ for $\mathrm{n}$ channel MOSFETs and $350 \mathrm{mV}$ for p-channel MOSFETs. Even safe experimental condition to perform circuit modification leads to unpredictable threshold shift accompanied by shifting of relevant transistor parameters including off-state leakage currents, and lower output current due to the decrease in mobility. To establish the focused ion beam as a potential circuit modification tool any unintentional change of the device performance or adjacent devices on the same die must be avoided. Clamping the transistor electrodes during exposure avoids any charging related device degradation. In order to quantify the extent of damage induced by FIB irradiation, during exposure the transistors are connected to a parameter analyzer. The output- and gate currents are monitored during trench milling.

The FIB-SEM image of the cross sectional view (Fig. 3) shows the V-shaped trench reaching $335 \mathrm{~nm}$ into the gate with a remaining gate seat of $S=165 \mathrm{~nm}$.

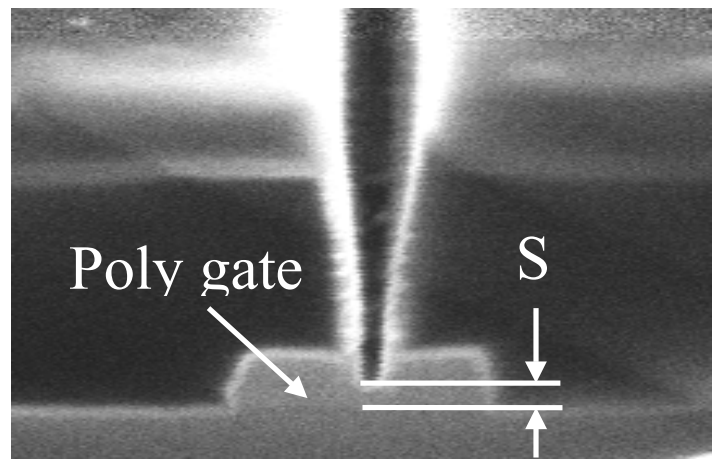

Figure 3. FIB-SEM image of the cross sectional view of the transistor with a trench reaching $335 \mathrm{~nm}$ into the poly gate.

Fig. 4 exhibits the output- and the gate current of the biased transistor as a function of milling depth as well as the residual distance to the gate oxide/bulk interface.

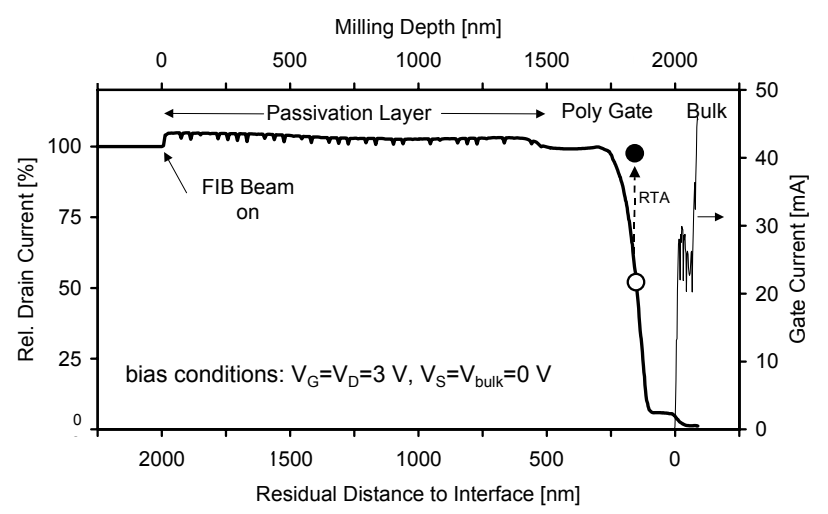

Figure 4. Output- and gate current of the n-MOS transistor vs. milling depth and recovery of output current after RTA.

The starting point of the milling process is accompanied by a tiny increase of the output current due to positive charging of the passivation layer. The periodic fluctuations of the drain current in this regime are a result of the milling strategy [5]. Every 6 seconds the beam is blanked, the charge deposited on the passivation tends to dissipate and the output current decreases. As long as the trench is located completely within the passivation layer, the transistor performance is not affected by the milling process. When the trench reaches the top of the gate electrode the conductive gate prevents further charging of the passivation and the drain current levels to the value of the undisturbed n-MOSFET. Apparent degradation starts as soon as long-range 
damage cascades extend into the channel region. These damage cascades reach into the gate oxide and have a finite chance to encounter an oxygen atom and knock it out of the gate oxide. Progressive FIB exposure leads to a loss of stoichiometric order of the oxide which results in the degradation of its dielectric properties [6]. Due to the poly- $\mathrm{Si} / \mathrm{SiO}_{2} / \mathrm{Si}$ system an additional degradation is expected from ion mixing of oxygen and silicon from both, the top poly-Si gatestack and underlying crystalline bulk. In case of the involved $50 \mathrm{keV}$ beam the drain current degrades if a residual gate stack of about $S=300$ $\mathrm{nm}$ is reached. The transistor with a residual gate seat of $\mathrm{S}=165 \mathrm{~nm}$ exhibits only half of the initial output current. Further milling leads to progressive device degradation until the onset of a gate current indicates a conductive path through the gate oxide.

Figure 5 shows the TEM image of the FIB modified transistor that exhibit $50 \%$ of the initial output current.

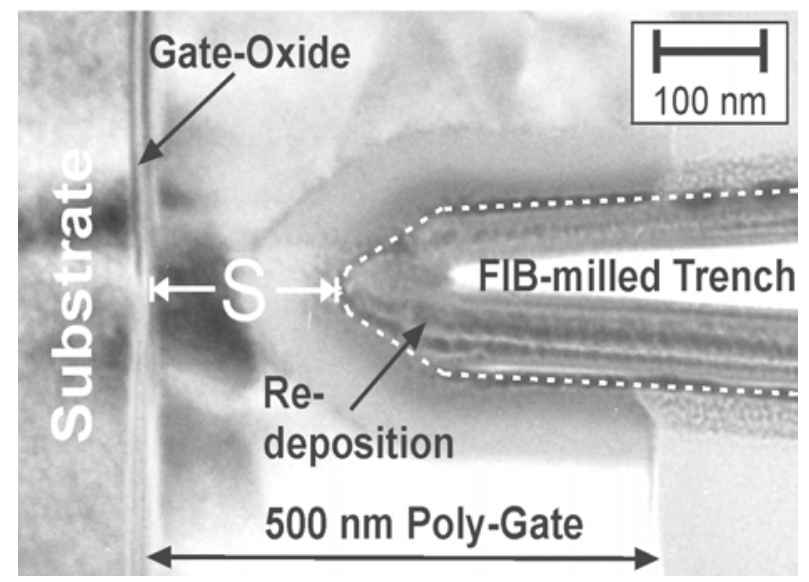

Figure 5. TEM (a) image of the cross sectional view of the transistor with a trench reaching $335 \mathrm{~nm}$ into the poly gate.

Even the extension of the amorphous region is kept fairly distant to the interface long-range damage cascades extend into the channel region and diminish the electron mobility in the disturbed channel region. Devices treated corresponding to this marked position in Fig. 4 could be fully recovered by a moderate RTA, indicated by the recovery of the drive current of the FIB modified transistors (full dot in Fig. 4). In order to demonstrate the feasibility of the FIB processing to front end modification, the $1,835 \mu \mathrm{m}$ deep trenches were employed as implantation windows. The through gate implantation of Ga respectively $\mathrm{P}$ and succeeding RTA at $600^{\circ} \mathrm{C}$ for $40 \mathrm{~s}$ yielding narrow $\mathrm{p}^{+}$respectively $\mathrm{n}^{+}$ regions in the channel.

Charge pumping was used to determine the interface state density and the residual damage after through-gate implantation and proper posttreatment. Figure 6 exhibits the efficiency of the RTA process for damage anneal.

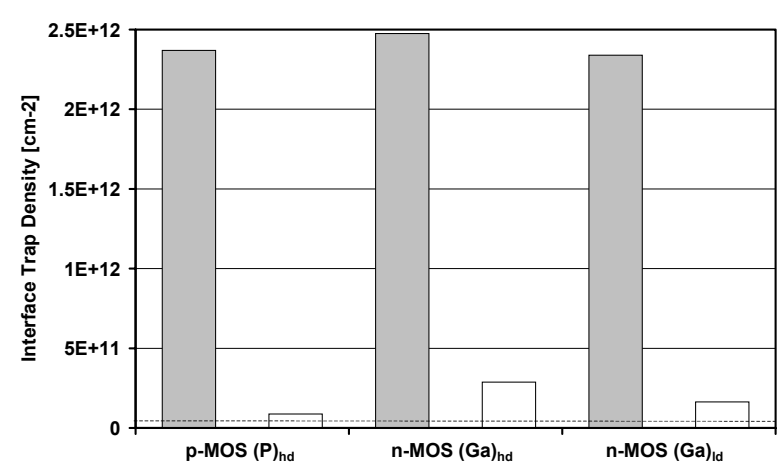

Figure 6. Interface trap density of MOSFET and FIB modified devices after through gate implantation (grey bars) and after RTA processing (white bars). The dashed line signs the interface state density of the conventional MOSFET devices.

As expected, the interface trap densities correlate with implantation doses and ion species, but the the RTA process reduces the defects to insignificant levels, comparable to conventional MOS devices.

Reducing the thermal budget to a single anneal and activation step, any unintentional change of the performance of neighboring devices could be avoided. The subthreshold characteristics in Fig. 7 exhibit regular behavior for the modified transistors.

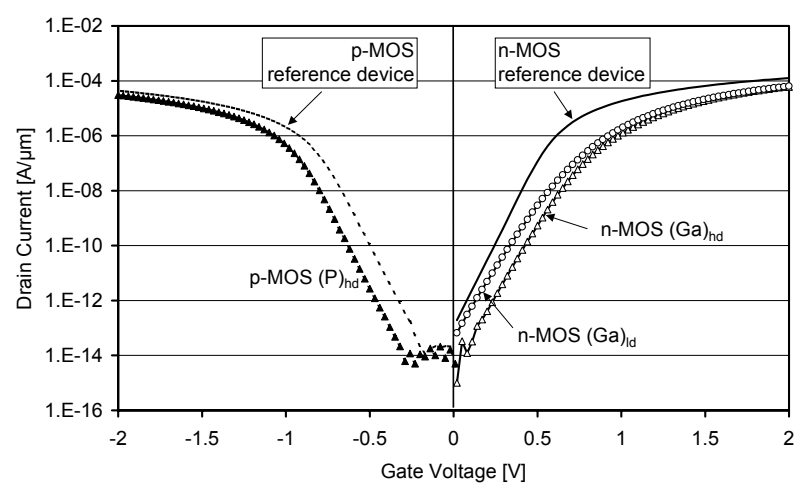

Figure 7. Comparison of the subthreshold characteristics for reference devices and modified $\mathrm{n}$ - and p-channel devices.

As expected the increase of the threshold voltage correlates with the implanted dose (Tab. 1). The minor threshold shift for p-channel devices might be a result of the lower degree of activation of $\mathrm{P}$ due to the moderate RTA. The off state leakage currents are one to two orders of magnitude lower compared to the reference device. Typical output characteristics of the modified transistors are compared to the reference MOSFET in Fig. 8. The roughly $30 \% \mathrm{Id}_{\text {sat }}$ degradation for the pchannel as well as the n-channel device can be attributed to the higher threshold voltage of the NUCMOS devices. 


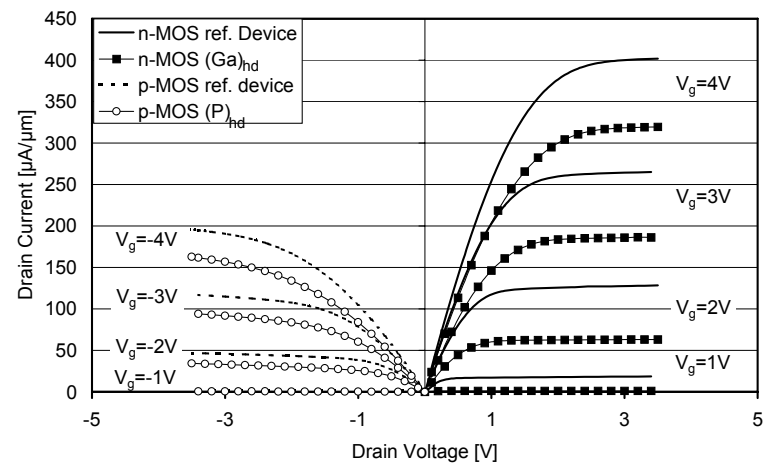

Figure 8. Transfer characteristics for conventional MOSFET and FIB modified $\mathrm{n}$ - and $\mathrm{p}$-channel devices

Table 1 exhibits the comparison of the main device parameters, including implantation dose and energy, threshold voltage and the subthreshold swing after annealing and reveal the good device performance.

TABLE 1. Device parameters: implantation dose $D$ and energy $E$, threshold voltage and subthreshold swing

\begin{tabular}{lccc}
\hline Device (dopant) & $\mathrm{D}\left[\right.$ ions $\left./ \mathrm{cm}^{2}\right] / \mathrm{E}[\mathrm{keV}]$ & $\begin{array}{c}\mathrm{V}_{\text {th }} \\
{[\mathrm{mV}]}\end{array}$ & $\begin{array}{c}\mathrm{SS} \\
{[\mathrm{mV} / \mathrm{dec}]}\end{array}$ \\
\hline n-MOS & none & 509 & 82 \\
n-MOS $(\mathrm{Ga})_{\mathrm{ld}}$ & $5 \cdot 10^{12} / 300$ & 842 & 103 \\
n-MOS $(\mathrm{Ga})_{\mathrm{hd}}$ & $2 \cdot 10^{13} / 300$ & 1018 & 98 \\
\hline p-MOS & none & -729 & 85,7 \\
p-MOS $(\mathrm{P})_{\text {hd }}$ & $2 \cdot 10^{13} / 180$ & -876 & 84.6 \\
\hline
\end{tabular}

\section{Summary}

This paper presents a novel technology of post-process CMOS front end engineering with FIB. The above results indicate that for the first time the focused ion beam responds to the demand for complex post process device modifications of fully featured wafer prepared by modern IC technology.

\section{Acknowledgements}

The authors would like to thank D. Schuhmann (Infineon) for providing the wafers, Ulf Grabner for TEM sample preparation and the austrian "Gesellschaft für Mikroelektronik" for financial support.

\section{References}

[1] J. Melngailis, C. R., Musil, E. H. Stevens, M. Utlaut, E. M. Kellog, R. T. Post, M. W. Geis, and R. W. Mountain, "The focused ion beam as an integrated circuit restructuring tool,” J. Vac. Sci. Technol. B., 4, 176 (1986).
[2] T. Lundquist, J. Brown, "Focused ion beams help debug analog and mixed-signal devices", Semiconductor-International. 19, no.10; 135, 138, 140, 142 (1996).

[3].J. Benbrik, P. Perdu, B. Benteo, R. Desplats, N. Labat, A. Touboul, Y. Danto, "Induced damages on CMOS and bipolar integrated structures under focused ion beam irradiation," Microelectr. Reliability, 38, 901 (1998).

[4].G. Groesenken, H. E. Maes, N. Beltran, R. F. de Keersmaecker, "A Reliable Approach to Charge Pumping Measurements in MOS Transistors," IEEE Transactions on Electron Devices, 31, 42 (1984).

[5] A. Lugstein, W. Brezna, and E. Bertagnolli, "Impact Of Focused Ion Beam Assisted Front End Processing on n-MOSFET Degradation", accepted for Int. Rel. Phys. Symp. (IRPS), (2002).

[6] M. G. Stinson, C. M. Osburn, "A knock-on model to explain enhanced perimeter leakage in ion-implanted metal-oxide semiconductor structures," J. Appl. Phys., 67, 4190 (1990). 